\title{
p53 suppresses stress-induced cellular senescence via regulation of autophagy under the deprivation of serum
}

\author{
XINBING SUI ${ }^{1,2^{*}}$, YONG FANG ${ }^{1,2}$, HAIZHOU LOU ${ }^{1,2}$, KAIFENG WANG $^{1,2}$, YU ZHENG $^{1,2}$, \\ FANG LOU ${ }^{1,2}$, WEI JIN ${ }^{1,2}$, YINGHUA XU ${ }^{1,2}$, WEI CHEN ${ }^{1,2}$, HONGMING PAN ${ }^{1,2}$, \\ $\mathrm{XIAN}_{\text {WANG }}^{1,2^{*}}$ and WEIDONG HAN ${ }^{1,2}$
}

${ }^{1}$ Department of Medical Oncology, and ${ }^{2}$ Biomedical Research Center and Key Laboratory of Biotherapy of Zhejiang Province, Sir Run Run Shaw Hospital, Zhejiang University, Hangzhou, Zhejiang 310016, P.R. China

Received February 7, 2014; Accepted September 24, 2014

DOI: $10.3892 / \mathrm{mmr} .2014 .2853$

\begin{abstract}
The tumor suppressor p53 is widely known for its ability to induce cell cycle arrest or cell death, therefore preventing neoplastic progression. Previous studies have demonstrated novel roles for p53 in the regulation of autophagy and senescence. p53 can not only exert cell cycle-arresting and senescence-promoting or suppressing functions, but can also induce autophagic flux, particularly under conditions of nutrient deprivation. The present study demonstrated that p53 was capable of activating autophagy, which permits cell survival under conditions of serun starvation, and suppresses cellular senescence through inhibition of the mammalian target of rapamycin pathway. These results suggest that active autophagy may be a potential mechanism by which p53 suppresses cellular senescence, in response to serum starvation. The findings of the present study provide a potential mechanism for suppression of senescence by $\mathrm{p} 53$

\section{Introduction}

The tumor suppressor p53, also known as the 'guardian of the cellular genome' and the 'cellular gatekeeper', is the most commonły mutated gene in human cancer $(1,2)$. p53 genetic or epigenetic inactivation has been reported in $\sim 50 \%$ of all human cancers (3). p53 as a tumor suppressor protein, has been shown to orchestrate a transcriptional stress response that may have numerous outcomes, including cell cycle arrest and apoptosis (4). Recent studies have
\end{abstract}

Correspondence to: Professor Hongming Pan or Dr Weidong Han, Department of Medical Oncology, Sir Run Run Shaw Hospital, Zhejiang University, 3 East Qingchuan Road, Hangzhou, Zhejiang 310016, P.R. China

E-mail: drpanhm@aliyun.com

E-mail: wangxzju@163.com

\section{*Contribued equally}

Key words: p53, autophagy, senescence, cell cycle, mTOR identified novel functions for $\mathrm{p} 53$, as a regulator of diverse biological processes, including metabolısm, autophagy and senescence (5-8).

Autophagy is an evolutionarily conserved catabolic process by which cytoplasmic constituents are sequestered within-autophagosomes, and targeted by lysosomes for digestion (9). Various stress signals can induce autophagy, including p53, nutrient deprivation, endoplasmic reticulum stress, hypoxia and other diverse stresses $(7,10,11)$. Autophagy may have either beneficial or detrimental cellular effects, depending on the response to environmental stresses. Increasing evidence suggests that autophagy facilitates cell survival by breaking down cytoplasmic components, in order to provide essential ingredients to maintain cellular metabolism under stressful conditions $(12,13)$. However, in some cases, autophagy may contribute to cell death by extensive digestion of intracellular organelles (14).

Cellular senescence is a process leading to irreversible arrest of the cell cycle and increased expression of $\beta$-galactosidase ( $\beta$-gal) activity at $\mathrm{pH} 6$. Senescence may be initiated by various insults, including telomere shortening (replicative senescence), oncogene activation, oxidative stress and DNA damage (15). Increasing evidence has shown that cellular senescence has an important role in the control of tumor progression, and by limiting cell proliferation it acts as an anticancer barrier $(16,17)$.

It has become increasingly apparent that p53 and the mammalian target of rapamycin (mTOR) pathway are critical mediators of the senescence response. p53 itself functions as either an activator or a repressor of senescence. p53 may induce senescence by activating downstream targets and preventing the block of the mTOR pathway (18). Conversely, in some cases, p53 may negatively regulate senescence by inhibiting the mTOR pathway (19). p53 regulation of senescence is dependent on the inverse correlation between p53 levels and mTOR activation, and both p53 and mTOR are also known for their autophagy-modulatory functions. However, it remains to be determined whether autophagic induction, in response to $\mathrm{p53}$, is associated with the regulation of senescence by $\mathrm{p} 53$.

The present study aimed to investigate the impact of autophagy, induced by p53, on cellular senescence. p53 was 
shown to suppress cellular senescence through the regulation of autophagy under serum-starved conditions.

\section{Materials and methods}

Cell culture and reagents. The HCT116, human colorectal carcinoma $\mathrm{p} 53^{+/+}$and $\mathrm{p} 53^{-/-}$cell lines were obtained from MD Anderson Cancer Center (Houston, TX, USA). All of the cell lines were maintained in McCoy's 5A media, supplemented with $10 \%$ fetal bovine serum, 100 units $/ \mathrm{ml}$ penicillin, $100 \mu \mathrm{g} / \mathrm{ml}$ streptomycin (Invitrogen Life Technologies, Carlsbad, CA, USA), and $2 \mathrm{mmol} / \mathrm{l} \mathrm{L-glutamine} \mathrm{in} \mathrm{a} \mathrm{humidified}$ atmosphere containing $95 \%$ air and $5 \% \mathrm{CO}_{2} .3$-Methyladenine (3-MA) was obtained from Sigma-Aldrich (St Louis, MO, USA) and was dissolved in heated sterile double distilled water, in order to produce a $400 \mathrm{mM}$ stock solution. The 3-MA stock solution was then added to the media, after heating, and a final concentration of $10 \mathrm{mM}$ was prepared $(20,21)$. Primary rabbit anti-LC3B $(1: 1,000)$ rabbit anti-p62 $(1: 1,000)$, rabbit anti-mTOR and anti-p-mTOR $(1: 1,000)$ and rabbit p-P70S6K $(1: 1,000)$ were purchased from Cell Signaling (Beverly, MA, USA), while mouse anti-p53 (1:500) and mouse anti-GAPDH (1:1,000) were purchased from Santa Cruz (Santa Cruz, CA, USA). The green fluorescent protein (GFP)-microtubule-associated protein 1 light chain 3 B (MAP1LC3B) plasmid was obtained from OriGene Technologies, Inc. (Rockville, MD, USA). Acridine orange solution (AO) was purchased from Sigma-Aldrich.

Measurement of cell viability. Cell viability was determiner by MTT assay (Sigma-Aldrich), light microscopy (Olymp Tokyo, Japan) and crystal violet staining (Sigma-Aldrich).

Cell cycle analysis. A fluorescence activated cerl sorting-assisted cell cycle analysis, for DNA content, was performed using the Cycletest ${ }^{\mathrm{TM}}$ Plus DNA Reagent kit (BD Biosciences, Franklin Lakes, NJ, USA), according to the manufacturer's instructions.

Detection of autophagy. To detect the presence of acidic vesicular organelles (AVO), the cells were stained for $24 \mathrm{~h}$ with the AO vital dye $(1 \mu \mathrm{g} / \mathrm{ml})$ and examined under a fluorescence microscope. GFP-MAP1LC3B transient transfection was performed according to the supplier's instructions. Briefly, sterile $12 \mathrm{~mm}$ cover slides were seeded with $5 \times 10^{5}$ cells into 6-well plates. After attachment overnight, cells were washed twice with phosphate-buffered saline (PBS) and the medium was replaced by serum-free medium for $24 \mathrm{~h}$ and then the cells were transiently transfected with a GFP-LC3B expressing vector (Origene, MD, USA). After $32 \mathrm{~h}$ of exposure to treatment, cells were washed with cold PBS and then fixed with $4 \%$ formaldehyde in PBS ( $\mathrm{pH}$ 7.4) for $20 \mathrm{~min}$ at room temperature.

$\beta$-gal staining. $\beta$-gal staining was performed using the Senescence-Galactosidase Staining kit (Cell Signaling Technology). After serum starvation for $24 \mathrm{~h}$, the cells were washed twice with PBS and then fixed with PBS containing $2 \%$ formaldehyde (Shanghai Biological Technology, Shanghai, China) and $0.2 \%$ glutaraldehyde (Shanghai Biological Technology) for $10 \mathrm{~min}$. The cells were then incubated at $37^{\circ} \mathrm{C}\left(\mathrm{NO} \mathrm{CO}_{2}\right)$ for $10 \mathrm{~h}$ with staining solution $(40 \mathrm{mM}$ citric acid sodium phosphate, $\mathrm{pH}$ 6.0, $1 \mathrm{mg} / \mathrm{ml}$ 5-bromo-4-chloro3-isolyl-B-D-galactoside [X-gal, Fisher, Pittsburgh, PA], 5 mM potassium ferricyanide, $150 \mathrm{mM} \mathrm{NaCl}, 2 \mathrm{mM} \mathrm{MgCl} 2$ ). After being washed twice with PBS, the cells were incubated with 4', 6-diamidino-2-phenlindole dihydrochloride (DAPI, $1 \mathrm{ug} / \mathrm{ml}$, DOJINDO, Tokyo, Japan) at $4^{\circ} \mathrm{C}$ for $2 \mathrm{~h}$. SA-B-gal-positive cells were enumerated by counting over 400 cells in three independent fields.

Western blotting. The cells were harvested from the $10 \mathrm{~cm}$ culture dishes via centrifugation $\left(800 \mathrm{x}\right.$ g for $30 \mathrm{~min}$ at $\left.4^{\circ} \mathrm{C}\right)$ and lysed in lysis buffer (20 mM Tris-HCl, pH 7.6; 1 mM EDTA; $140 \mathrm{mM} \mathrm{NaCl} ; 1 \% \mathrm{NP}-40 ; 1 \%$ aprotinin; $1 \mathrm{mM}$ phenylmethylsulfonyl fluoride; $1 \mathrm{mM}$ sodium vanadate). The protein concentrations were determined using a Bicinchoninic Acid Protein Assay kit (Pierce Biotechnology, Inc., Rockford, IL, USA). The cell lysates (40 $\mu$ g protein/lane) were loaded onto 5-20\% Tris-Tricine Ready SDS-PAGE gels (Bio-Rad Laboratories, Inc., Hercules, CA, USA), separated by electrophoresis, and transferred to nitrocellulose membranes (ShanghaiZDAN International, Shanghai, China). The blotted membranes were blocked with $5 \%$ skim milk for $1 \mathrm{~h}$ and were then-incubated with the primary antibodies for $2 \mathrm{~h}$ at $37^{\circ} \mathrm{C}$. The immunoreactive bands were visualized by enhanced chemiluminescence (Pierce, Rockford, IL, USA), following a further incubation with horseradish peroxidase-conjugated immunoglobulin $\mathrm{G}$ secondary antibodies. The band density was determined by densitometry, and quantified using gel plotting macros of National Institutes of Health (NIH) Image 1.62, (NH, Bethesda, MA, USA).

Cell cycle analysis. Cell cycle distribution was determined by flow cytometry. Briefly, a total of $1-2 \times 10^{6}$ cells were cultured in medium containing $10 \%$ FBS. Serum was withdrawn from culture medium when cells were $70 \%$ confluent. After $72 \mathrm{~h}$, $10 \%$ FBS was added to the medium for an additional $12 \mathrm{~h}$. Cells were fixed in $70 \%$ ethanol and stained with propidium iodide (BD Pharmingen, San Jose, CA) and DNA content was analysed by FACSCalibur Flow Cytometer (BD Biosystems, Heidelberg, Germany).

Statistical analysis. The results in the figures are presented as the mean \pm standard deviation. Statistical analysis was performed in SPSS version 11.0 for Windows (SPSS, Inc., Chicago, IL, USA).

\section{Results}

p53 promotes HCT116 cell survival by inducing autophagyunder the deprivation of serum. To examine the impact of p53 on cell survival, following a $24 \mathrm{~h}$ period of serum starvation, the human colorectal carcinoma-derived HCT116 cells with wild-type p53 (HCT116 p53 $3^{+/}$) were compared with their isogenic derivatives, in which the p53 gene had been somatically knocked out $\left(\mathrm{HCT} 116 \mathrm{p} 53^{-/}\right)$. The levels of autophagic flux were compared in the HCT116 p53 ${ }^{+/+}$and HCT116 p53 ${ }^{-/}$cells under normal conditions (Cont), serum starvation (Stv. $24 \mathrm{~h}$ ) and serum starvation in the presence of an inhibitor of autophagy, 3-MA (Stv. $24 \mathrm{~h}+$ 3-MA), for $24 \mathrm{~h}$. The levels of autophagy were analyzed in both

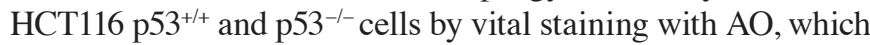


A

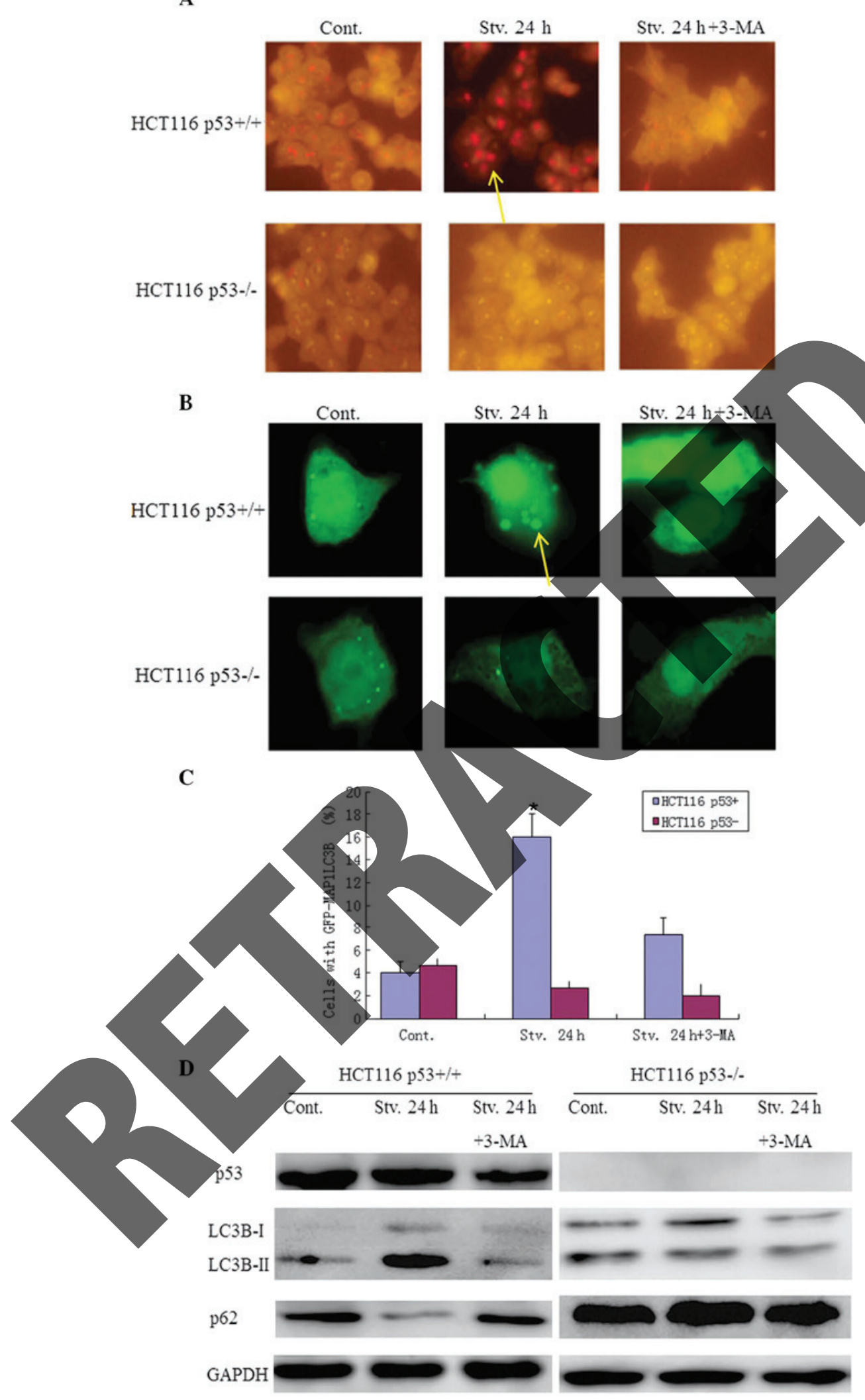

Figure 1. p53 activates autophagic flux in response to serum starvation. (A) Acridine orange (AO) staining. The cells were stained with the vital dye $\mathrm{AO}(1 \mu \mathrm{g} / \mathrm{ml})$ and examined under a fluorescence microscope (magnification, x20). (B) (Top) Green fluorescent protein (GFP)-microtubule-associated protein 1 light chain 3 B (MAP1LC3B) was transiently transfected into HCT116 human colorectal cancer cells. p53 $3^{+/+}$and p53 $3^{-/-}$cells were maintained under normal conditions, serum starvation or serum starvation in the presence of 3-methyladenine (3-MA), for $24 \mathrm{~h}$. The cells were washed with cold phosphate-buffered saline (PBS) and fixed with $4 \%$ formaldehyde in PBS (pH 7.4), for $20 \mathrm{~min}$ at room temperature, and then observed under a fluorescence microscope (magnification, x10). (Bottom) Autophagic flux was quantified by counting the percentage of cells showing the accumulation of GFP-MAP1LC3B in aggregates or vacuoles. The data represent the means \pm standard deviation. (C) Quantitative analysis of GFP-MAP1LC3B. The values represent the means \pm standard deviation of three independent experiments; "P<0.05. (D) After $24 \mathrm{~h}$, the cells were lysed and western blotting was performed with a LC3B antibody. GAPDH was used as a loading control. Cont, control; Stv, serum starvation; $553^{+/+}$cells with wild-type $\mathrm{p} 53$; $\mathrm{p} 53^{-/}$cells with a somatic knockout of p53 expression. Yellow arrows: punctate fluorescence. 
A
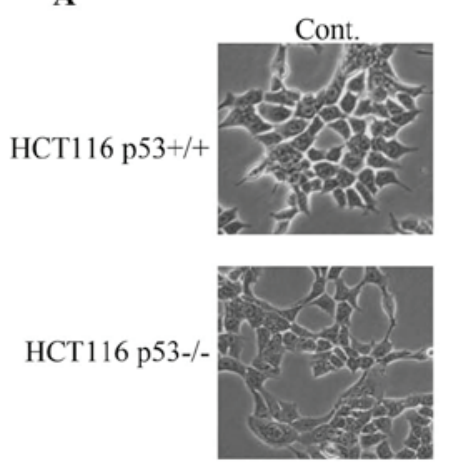

Stv. $24 \mathrm{~h}$
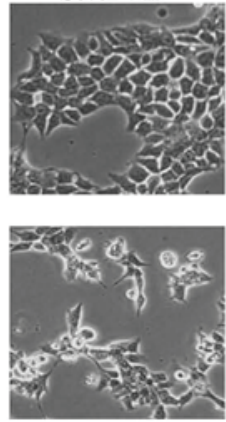

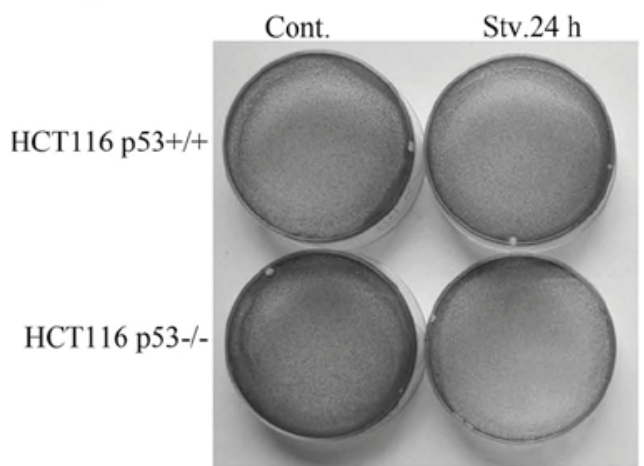

C

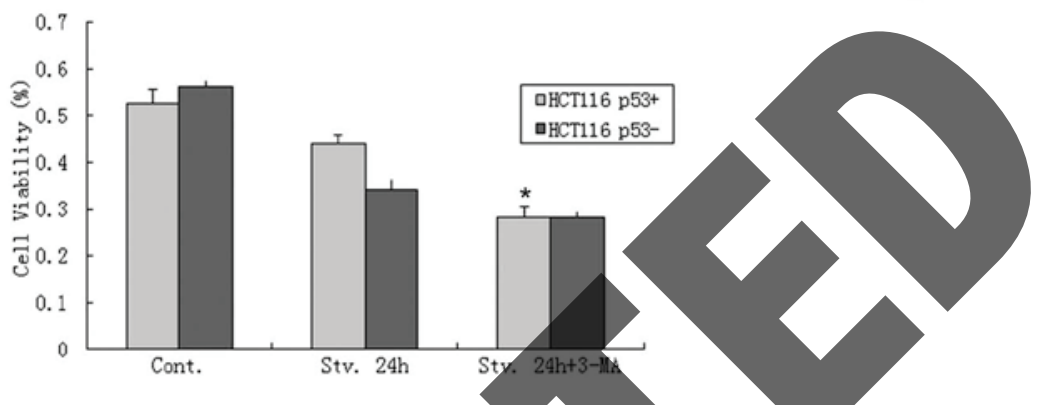

Figure 2. Autophagy induced by p53 maintains HCT116 human colorectal carcinoma cell survival under conditions of serum starvation. (A) p53 $3^{+/+}$and p53 $3^{-/-}$ cells were maintained under normal conditions or serum starvation for $24 \mathrm{~h}$, and /ive cell images were captured (magnification, x10). (B) Following a $24 \mathrm{~h}$ culture, the cells were fixed and stained with crystal violet (magnification, x10). (C) The results from the MTT assay. The cells were seeded in 96-well flat bottom microtiter plates at a density of $1 \times 10^{4}$ cells/well. Following a 24 culture the absorbance was measured at $570 \mathrm{~nm}$ using a microplate reader (Synergy

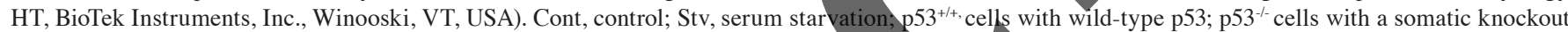
of p53 expression. ${ }^{*} \mathrm{P}<0.05$ compared withj control.

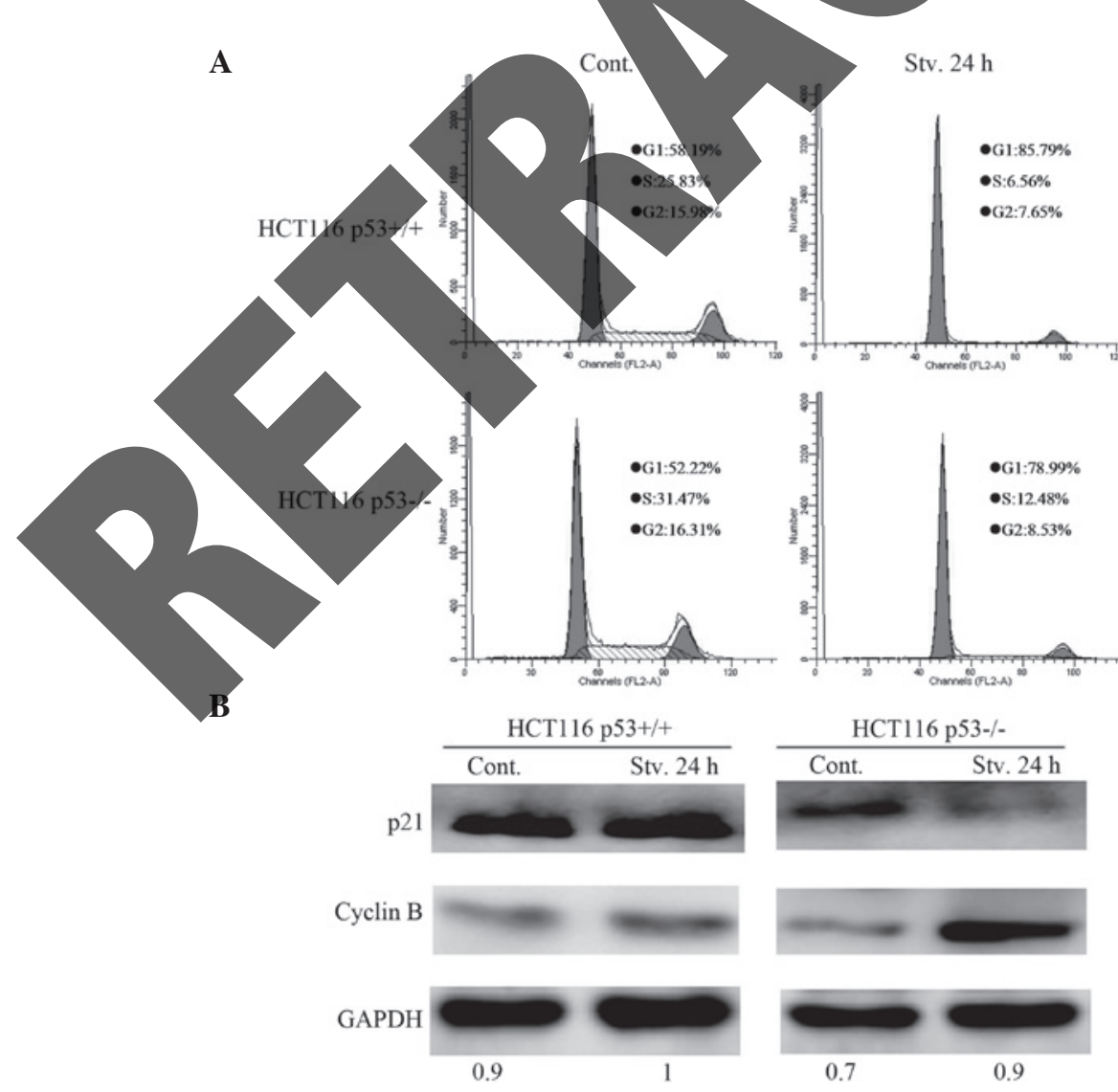

Figure 3. Effects of p53 on the starvation-induced cell cycle. (A) Following a $24 \mathrm{~h}$ exposure to treatment, the HCT116 human colorectal carcinoma cells were trypsinized, stained with propidium iodide, and analyzed using a FACSsort flow cytometer. (B) Following a $24 \mathrm{~h}$ exposure to treatment, the cells were lysed and western blotting was conducted using p21 and cyclin B antibodies. The expression of GAPDH in (HCT116 p53-/- Cont.)/(HCT116 p53+/+ (Stv.)=0.7; the expression of GAPDH in (HCT116 p53-/- Stv.) or (HCT116 p53+/+ Cont.)/(HCT116 p53+/+ Stv.)=0.9. Cont, control; Stv, serum starvation; p53 ${ }^{+/+}$cells with wild-type 553 ; $53^{-1-}$ cells with a somatic knockout of p53 expression. 
A
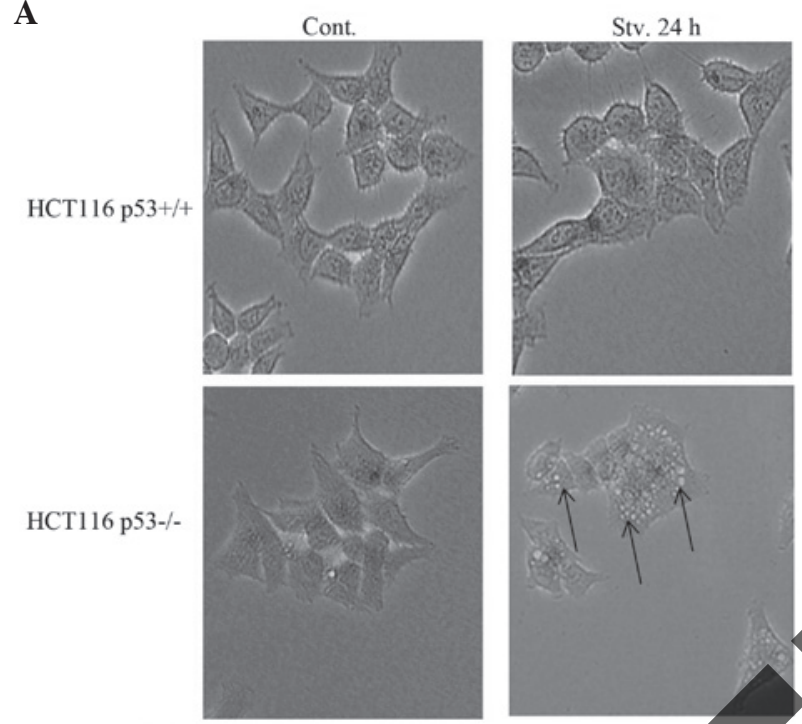

B
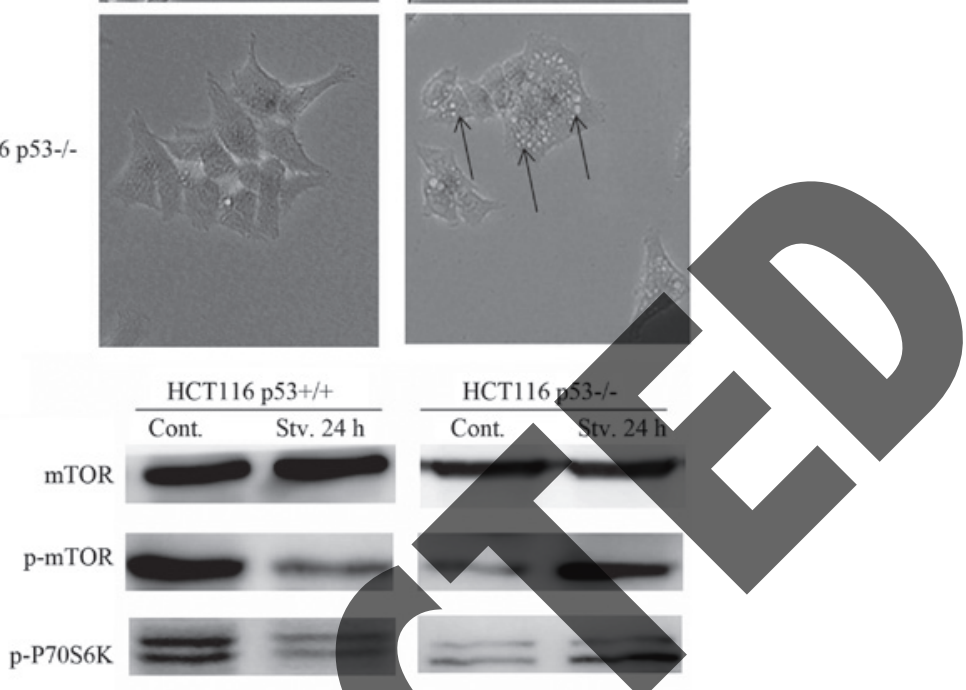

GAPDH

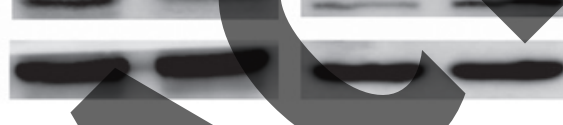

Figure 4. Effects of p53-dependent autophagy on cellular senescence and the regulation of phosphatase and tensin homolog. (A) p53 $3^{+/+}$and p53 ${ }^{-/-}$HCT116 human colorectal carcinoma cells were maintained under normal conditions, serum starvation or serum starvation in the presence of 3-methyladenine (3-MA) for $24 \mathrm{~h}$. All cells were stained for $\beta$-galactosidase, to indieate senescence (magnification, x20). (B) The expression levels of mammalian target of rapamycin (mTOR), phospho(p)-mTOR and phospho-P70S6K in the $\mathrm{p} 53^{+/+}$and $\mathrm{p} 53^{-/}$cells exposed to treatment for $24 \mathrm{~h}$, were analyzed by western blotting. Cont, control; Stv, serum starvation; $\mathrm{p} 53^{+/+}$cells with wild-type $\mathrm{p} 53, \mathrm{p} 53^{-1-}$ cells with a somatic knockout of $\mathrm{p} 53$ expression.

stains AVOs, including autophagic vacuoles. An increase in the intensity of AVOs was observed by fluorescent microscopy in the HCT116 $\mathrm{p}^{+/++}$cells following serum starvation, however few AVOs were detected in the HCT116 $553^{--}$cells (Fig. 1A). These results indicate that 53 may activate autophagy under serum starvation. Furthermore, autøphagie flux induced by p53 was attenuated by treatment with $10 \mathrm{mmol} / \mathrm{h}$-MA (Fig. 1A). For further confirmation, the HCT116 eells were transiently transfected with a GFP-MAPILC3B plasmid. MAP1LC3B is a key component of the autophagosomal membrane, and is associated with autophagosome generation (22). Upon induction of autophagy, punctate fluorescence of GFP-MAP1LC3B can be observed. Punctate fluorescence was markedly evident in the HCT116 $\mathrm{p} 53^{+/+}$cells, as compared with the $\mathrm{p} 53^{-/-}$cells, following serum starvation, and this fluorescence was effectively inhibited by 3-MA (Fig. 1B). These results further imply that p53 is capable of activating autophagy under serum-starved conditions. In addition, the western blot analysis (Fig. 1C) detected a significantly increased LC3-II/I ratio, and decreased abundance of $\mathrm{p} 62$ in the $\mathrm{p} 53^{+/+}$cells, as compared with the $\mathrm{p} 53^{-/-}$ cells following serum starvation. These data indicate that $\mathrm{p} 53$ promotes autophagy and suggest that p 53 may induce autophagy under serum-starved conditions.

It is well known that p53 can promote apoptosis under normal conditions (Fig. 2); however, the present study aimed to determine whether $\mathrm{p} 53$ could still induce apoptosis in response to serum starvation. The impact of autophagy, induced by p53 under serum starvation, was assessed on cell survival using an MTT assay, light microscopy and crystal violet staining. Notably, more viable cells were detected in the HCT116 $\mathrm{p} 53^{+/+}$group as compared with the HCT116 $\mathrm{p} 53^{-/}$group following serum starvation for $24 \mathrm{~h}$ (Fig. 2A-C). These results imply that p53 may facilitate cellular survival in response to serum starvation. To determine whether p53-dependent autophagic regulation protects cancer cells from starvation-induced death, cell viability was determined in both $\mathrm{HCT} 116 \mathrm{p} 53^{+/+}$and $\mathrm{p} 53^{-/-}$cells following serum starvation, in the presence of 3-MA.

A large number of apoptotic cells were present in the $\mathrm{p} 53^{+/+}$group following treatment with 3-MA, as determined by flow cytometry; however, no significant changes were observed in the apoptosis of the p53 $3^{--}$cells (Fig. 2C). These results indicate that $\mathrm{p} 53$-dependent autophagic regulation

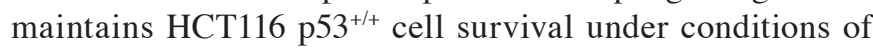
serum starvation.

p53 suppresses stress-induced senescence and simultaneously causes quiescence in HCT116 cancer cells. Cellular senescence is a process that leads to the irreversible loss of proliferative potential, and permanent growth arrest. Quiescence is significantly different to senescence, as it results in a stable but reversible growth arrest (23). Previous 
studies have suggested that p53 promotes senescence, by p53-induced cell cycle arrest $(15,16,24)$. Furthermore, p53, as a transcriptional regulator, may induce transcription of cyclin-dependent kinase inhibitor p21, which is upregulated in numerous cell lines during senescence and cell cycle arrest $(25,26)$. However, recent studies have shown that p53 may also negatively regulate senescence $(19,23,27)$. Therefore, the effects of p53 on the starvation-induced cell cycle were assessed in the present study, by propidium iodide staining and subsequent flow cytometric analysis. p53 inhibited the $\mathrm{G}_{1}$-to-S transitions: $\mathrm{G}_{1} / \mathrm{S} 13.08$ in $\mathrm{p}^{+/+}$cells, as compared with only 6.33 in $\mathrm{p}^{2} 3^{-/}$cells (Fig. 3A). These results demonstrate that p53 may promote starvation-induced cell cycle arrest. Western blotting showed that the expression of p21 was suppressed, and the protein expression levels of cyclin B were enhanced in the starved HCT116 p53 ${ }^{-/}$cells, as compared with the $\mathrm{p} 53^{+/+}$cells (Fig. 3B), thus implying that p53 inhibits starvation-induced cell cycle progression. These data suggest that p53 may induce cell cycle arrest in response to serum starvation, thus resulting in cell quiescence.

To confirm the role of p53 in cellular senescence in response to serum starvation, staining with $\beta$-gal was conducted. $\beta$-gal is a well-known marker present only in senescent cells, but not in pre-senescent, quiescent or immortal cells. $\beta$-gal activity was significantly increased in

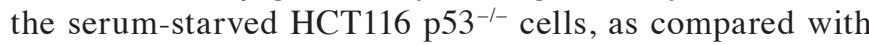
the serum-starved HCT116 p53 $3^{+/+}$cells (Fig. 4A), suggesting that p53 may function as a suppressor of cellular senescenc in response to serum starvation. These data suggest tha although p53 induces the expression of p21 and causes cell cycle arrest in the absence of serum, p53 simultaneously suppresses, rather than promotes stress-induced senescence. p53 suppresses cellular senescence through regulation of autophagy. In the absence of serum, p53 may activate autophagic flux, which can be attenuated by treatment with 3-MA. The present study aimed to determine whether inhibition of p53-dependent autophagy may promote cellular senescence. Both starved HCT116 $\mathrm{p53}^{+/+}$and $\mathrm{p53}^{->}$cells were treated with 3-MA. $\beta$-gal activity was markedly enhanced in the starved HCT116 $\mathrm{p}^{+/+}$cells in response to 3-MA treatment, but hardly changed in the starved $553^{-1-}$ cells (Fig. 4A). These results indicate that inhibition of $\mathrm{p53}$-dependent autophagy may promote cellular senescence

To determine the potential mechanisms by which p53-dependent autophagy suppresses senescence, the impact of autophagy induced by p53 was observed on the mTOR pathway, which is a contributor to cellular senescence. mTOR acts as a key regulator during cellular processes such as cell-cycle progression, anabolism, autophagy and senescence (28). The maintenance of mTOR signaling under conditions of cell cycle arrest may lead to senescence, whereas inhibition of mTOR by rapamycin converts senescence into quiescence (29). Another response to mTOR inhibition is the induction of autophagy, which allows cell survival under conditions of nutrient deprivation. p53 is able to inhibit the mTOR pathway through a number of known p53 transcriptional targets, including AMP-activated protein kinase, tuberous sclerosis complex 2, phosphatase and tensin homolog (PTEN), and sestrins, all of which are upstream negative regulators of the mTOR pathway $(30,31)$. Therefore, the present study examined whether p53-dependent autophagy was capable of suppressing senescence through the inhibition of mTOR. The expression levels of mTOR, phospho-mTOR and phospho-P70S6K were determined. Under the deprivation of serum, mTOR and P70S6K phosphorylation were significantly inhibited in the HCT116 p53 $3^{+/+}$cells (Fig. 4A and B). These results suggest that suppression of senescence through p53-dependent autophagy may occur partly through the inhibition of the mTOR pathway.

\section{Discussion}

Previous studies have implicated p53 in the promotion of senescence; however, a controversial role of p53 in the regulation of senescence has emerged in recent studies (8-15). p53 has been shown to inhibit senescence, while promoting cell cycle arrest (19). The subsequent fate of the arrested cells is considered to be dependent on p53-associated regulation of the mTOR pathway, active mTOR leads to senescence, whereas inhibition of mTOR suppresses senescence and leads to quiescence $(27,29)$

Despite the conflicting evidence regarding the ability of p53 to enhance or inhibit autophagy (32), the present study showed that p53 may promote autophagic flux, in response to serum starvation. Active autophagy allows cells to break down long-lived proteins and organelles, thus providing metabolites and ATP that may be used for cell survival, in order to cope with diverse environmental stresses (12). Furthermore, autophagy inctuced by p53 may be inhibited through enhancement of mTOR signaling, resulting in a marked increase in the levels of cell death in the starved HCT116 p53 ${ }^{+/+}$cells, following treatment with 3-MA.

The principal role of p53 is to promote p21-dependent cell cycle arrest; however, subsequent overexpression of p21 can not induce senescence since the transcriptional level of p21 is abrogated in senescent HCT116 p53-/- cells. This implies that p21 is not the determinant of cellular senescence. The present study determined the expression levels of PTEN, a negative regulator of the mTOR pathway. The results demonstrated that activation of mTOR is required for induction of senescence, not $\mathrm{p} 21$.

p53 has previously been shown to be capable of inhibiting the mTOR pathway at numerous levels in response to stress $(30,33)$, and mTOR inhibition may promote autophagy and suppress cellular senescence. However, there is little knowledge regarding p53-induced autophagy and its involvement in cellular senescence. Therefore, the present study tested the hypothesis that inhibition of autophagy by 3-MA treatment contributed to cellular senescence. 3-MA is an inhibitor of phosphatidylinositol 3-kinase and is widely used to suppress autophagy. In agreement with the hypothesis, p53 suppressed cellular senescence under serum starvation conditions, which may be attributed to active autophagy induced by p53 (11). In the presence of 3-MA, senescent morphologies were evident

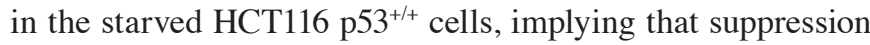
of senescence by p53 may be associated with the regulation of p53-dependent autophagy. Furthermore, this phenomenon may be explained by the regulation of the mTOR pathway.

In conclusion, the results of the present study suggest that, in response to serum starvation, p53 activates autophagic flux 
and subsequent autophagy functions as a survival pathway, in order to suppress cellular senescence by regulation of the mTOR pathway. This provides a potential association between autophagy and senescence and explains why p53 may suppress cellular senescence in response to starvation.

\section{Acknowledgements}

The authors of the present study would like to thank Mian $\mathrm{Wu}$, for providing the HCT116 $\mathrm{p} 53^{+/+}$and $\mathrm{p} 53^{-/-}$cell lines. The present study was supported by grants from the National Natural Science Foundation of China (nos. 81301891, 81272593, 81071651 and 81071963) and the Zhejiang Provincial Natural Science Foundation of China (nos. LQ13H160008 and LQ13H160009).

\section{References}

1. Lane DP: Cancer. p53, guardian of the genome. Nature 358: $15-16,1992$.

2. Levine AJ: p53, the cellular gatekeeper for growth and division. Cell 88: 323-331, 1997.

3. Soussi $\mathrm{T}$ and Wiman KG: Shaping genetic alterations in human cancer: the p53 mutation paradigm. Cancer Cell 12: 303-312, 2007.

4. Zilfou JT and Lowe SW: Tumor suppressive functions of p53. Cold Spring Harb Perspect Biol 1: a001883, 2009.

5. Vousden KH and Ryan KM: p53 and metabolism. Nat Rev Cancer 9: 691-700, 2009.

6. Abida WM and Gu W: p53-Dependent and p53-independent activation of autophagy by ARF. Cancer Res 68: 352-357,20

7. Tasdemir E, Maiuri MC, Galluzzi L, et al: Regulation autophagy by cytoplasmic p53. Nat Cell Biol 10: 676-687, 20

8. Xue W, Zender L, Miething C, et al: Senescence and tumour clearance is triggered by $\mathrm{p} 53$ restoration in murine live carcinomas. Nature 445: 656-660, 2007.

9. Sui X, Jin L, Huang X, Geng S, He C and Hu X: p53 signaling and autophagy in cancer: a revolutionary strategy could be developed for cancer treatment. Autophagy 7: 565-571, 2011

10. Kondo Y, Kanzawa T, Sawaya R and Kondo S: The role of autophagy in cancer development and response to therapy. Nat Rev Cancer 5: 726-734, 2005

11. Yang Z and Klionsky DJ: An overyiew of the molecular mechanism of autophagy. Curr Top Microbiol Immunol 335: 1-32, 2009.

12. Lum JJ, DeBerardinis RJ and Thompson CB: Autophagy in metazoans: cell survival in the land of plenty. Nat Rev Mol Cell Biol 6: 439-448, 2005

13. Scherz-Shouval R, Weldberg H, Gonen C, Wilder S, Elazar Z and Oren $\mathrm{M}$ : p53-dependent regulation of autophagy protein LC3 supports cancer cell survival under prolonged starvation. Proc Natl Acad Sci USA 107: 18511-18516,2010.

14. Mizushima N, Levine B, Cuervo AM and Klionsky DJ: Autophagy fights disease through cellular self-digestion. Nature 451: 1069-1075, 2008
15. Chen Z, Trotman LC, Shaffer D, et al: Crucial role of p53-dependent cellular senescence in suppression of Pten-deficient tumorigenesis. Nature 436: 725-730, 2005.

16. Ferbeyre G, de Stanchina E, Lin AW, et al: Oncogenic ras and p53 cooperate to induce cellular senescence. Mol Cell Biol 22: 3497-3508, 2002.

17. Lin HK, Chen Z, Wang G, et al: Skp2 targeting suppresses tumorigenesis by Arf-p53-independent cellular senescence. Nature 464: 374-379, 2010.

18. Santoro R and Blandino G: p53: The pivot between cell cycle arrest and senescence. Cell Cycle 9: 4262-4263, 2010.

19. Demidenko ZN, Korotchkina LG, Gudkov AV and Blagosklonny MV: Paradoxical suppression of cellular senescence by p53. Proc Natl Acad Sci USA 107: 9660-9664, 2010.

20. Pan H, Wang Z, Jiang L, et al: Autophagy inhibition sensitizes hepatocellular carcinoma to the multikinase inhibitor linifanib. Sci Rep 4: 6683, 2014.

21. Sui X, Kong N, Wang X, et al: JNK confers 5-fluorouracil resistance in p53-expressing colon cancer cells by inducing survival autophagy. Sci Rep 4: 4694, 2014

22. Rzymski T, Milani M,Pike L, et al: Regulation of autophagy by ATF4 in response to severe hypoxia. Oncogene 29: 4424-4435, 2010.

23. Serrano M: Shifting senescence into quiescence by turning up p53. Cell Cycle 9: 4256-4257, 2010.

24. Fujita K, Horikawa I, Mondal AM, et al: Positive feedback between $\mathrm{p} 53$ and TRF2 during telomere-damage signaling and cellular senescence. Nat Cell Biol 12: 1205-1212, 2010.

25. Alcorta DA, Xiong Y, Phelps D, Hannon G, Beach D and Barrett JC: Involvement of the cyclin-dependent kinase inhibitor p16 (INK4a) in replicative senescence of normal human fibrolasts. Proc Natl Acad Sci USA 93: 13742- 13747, 1996.

26. Kang JY, Kim JJ, Jang SY Y and Bae YS: The p53-p21(Cip1/WAF1) athway is necessary for cellular senescence induced by the inhibition of protein kinase CKII in human colon cancer cells. Mol Cells 28: 489-494, 2009

Korotchkina LG, Leontieva OV, Bukreeva EI, Demidenko ZN, Gudkov AV and Blagosklonny MV: The choice between p53-induced senescence and quiescence is determined in part by the mTOR pathway. Aging (Albany NY) 2: 344-352, 2010.

. Zoncu R, Efeyan A and Sabatini DM: mTOR: from growth signal integration to cancer, diabetes and ageing. Nat Rev Mol Cell Biol 12: 21-35, 2011.

29. Demidenko ZN and Blagosklonny MV: Growth stimulation leads to cellular senescence when the cell cycle is blocked. Cell Cycle 7: 3355-3361, 2008.

30. Feng $\mathrm{Z}, \mathrm{Hu} \mathrm{W}$, de Stanchina $\mathrm{E}$, et al: The regulation of AMPK beta1, TSC2, and PTEN expression by p53: stress, cell and tissue specificity, and the role of these gene products in modulating the IGF-1-AKT-mTOR pathways. Cancer Res 67: 3043-3053, 2007.

31. Budanov AV and Karin M: p53 target genes sestrin1 and sestrin2 connect genotoxic stress and mTOR signaling. Cell 134: 451-460, 2008.

32. Levine B and Abrams J: p53: The Janus of autophagy? Nat Cell Biol 10: 637-639, 2008.

33. Feng $\mathrm{Z}$ and Levine AJ: The regulation of energy metabolism and the IGF-1/mTOR pathways by the p53 protein. Trends Cell Biol 20: 427-434, 2010. 